Disponível em

http://www.anpad.org.br/rac

RAC, Rio de Janeiro, v. 18, n. 2, art. 3, pp. 161-175, Mar./Abr. 2014

$(\infty)$ EY-No

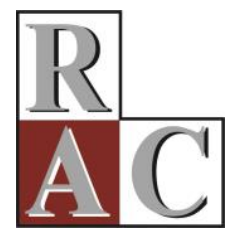

\title{
Relações entre Resistência a Mudança e Comprometimento Organizacional em Servidores Públicos de Minas Gerais
}

\section{Relationships between Resistance to Change and Organizational Commitment of Public Employees from Minas Gerais}

Antônio Luiz Marques

E-mail: marques@ face.ufmg.br

Universidade Federal de Minas Gerais - CEPEAD/UFMG CEPEAD/UFMG, Av. Antonio Carlos, 6627, Sala 4053, 31270-901, Belo Horizonte, MG, Brasil.

Renata Borges

E-mail: renatasg@face.ufmg.br

Universidade Federal de Minas Gerais - CEPEAD/UFMG CEPEAD/UFMG, Av. Antonio Carlos, 6627, Sala 4067, 31270-901, Belo Horizonte, MG, Brasil.

Kelly Morais

E-mail: kellyadm2@gmail.com Universidade Federal de Minas Gerais - CEPEAD/UFMG CEPEAD/UFMG, Av. Antonio Carlos, 6627, Sala 4121, 31270-901, Belo Horizonte, MG, Brasil.

Mariane Coimbra Silva

E-mail: marianecoimbrasilva@gmail.com Universidade Federal de Minas Gerais - CEPEAD/UFMG CEPEAD/UFMG, Av. Antonio Carlos, 6627, Sala 4421, 31270-901, Belo Horizonte, MG, Brasil. 


\title{
Resumo
}

O objetivo desta pesquisa é identificar as possíveis relações entre resistência à mudança e comprometimento organizacional. Neste estudo, foram avaliados servidores públicos do estado de Minas Gerais submetidos à Avaliação de Desempenho Individual (ADI), ferramenta implantada pelo governo mineiro junto a diversas mudanças que fazem parte do projeto Choque de Gestão. Para testar a hipótese da pesquisa, foi realizado um survey em três Secretarias do Estado de Minas Gerais, cuja amostra obtida foi de 679 respondentes. Utilizou-se a análise fatorial para avaliar o instrumento utilizado, empregou-se a análise de variância para identificar a diferença entre as secretarias de Estado e a regressão linear para testar a hipótese proposta. Os resultados indicam que uma variação positiva da aceitação e da cooperação com a implantação da ADI resulta em aumento do comprometimento percebido, ou seja, o servidor que coopera com as mudanças implantadas tende a apresentar um alto comprometimento com a organização. A principal contribuição da pesquisa é, portanto, relacionar o comprometimento com a percepção que os servidores têm sobre as mudanças organizacionais implantadas pelo governo de Minas Gerais.

Palavras-chave: mudança organizacional; comprometimento no trabalho; avaliação de desempenho.

\begin{abstract}
The objective of this research is to analyze the relationship between resistance to change and organizational commitment of public employees from the state of Minas Gerais in Brazil. This study includes only public employees who participated in the individual performance appraisal program (ADI), which was developed by the state government along with other changes integrating the "Management Shock" project. A survey method was employed to test the hypothesis. The sample size is 679 respondents from three State Secretary offices of Minas Gerais. Factorial analysis was preferred to estimate the measurement model, linear regression was employed to test the hypothesis, and variance analysis was run to test the differences between the Secretaries of State. The results indicate that a positive variation in the acceptance of and cooperation with the individual performance appraisal program leads to an increase in organizational commitment level. In other words, employees who collaborate with the changes are more likely to be highly committed to the organization. The main contribution of this research is to relate commitment to the perception of organizational changes employees experience as result of the management shock program.
\end{abstract}

Key words: organizational change; organizational commitment; performance appraisal. 


\section{Introdução}

Um dos maiores desafios dos gerentes e gestores de recursos humanos na atualidade é transformar o capital humano de suas empresas em vantagem competitiva, para que elas possam apresentar bom desempenho em um ambiente em transformação, envolvendo rápidas mudanças (Jones, 2007). A sobrevivência das organizações tem sido apontada como altamente dependente da capacidade de elas próprias, continuamente, modificarem-se e reinventarem-se (Kotter, 1995). No entanto, mudanças trazem incertezas e turbulências no ambiente organizacional (Elias, 2009; Peccei, Giangreco, \& Sebastiano, 2011) que podem gerar resistências decorrentes da necessidade implicada aos trabalhadores de sair de uma situação conhecida para uma situação desconhecida (Coghlan, 1993; Nadler, 1981; Steinburg, 1992).

Estudos anteriores concluíram que a mudança, especialmente a resistência à mudança, afeta negativamente o comprometimento dos trabalhadores (Fedor, Caldwell, \& Herold, 2006; G. E. Lambert \& Hogan, 2010; Marques, Borges, \& Carvalho-Freitas, 2007). O termo comprometimento, do ponto de vista científico, refere-se a uma "adesão, um forte envolvimento do indivíduo com vários aspectos do ambiente de trabalho" (Bastos, 1995, p. 46). Dessa forma, o comprometimento tem sido apontado como tendo uma relação inversa com absenteísmo e turnover, sendo que, quanto maior o nível de comprometimento, menor a frequência desses fatores que prejudicam o desempenho organizacional (E. I. Lambert, 2006; G. E. Lambert \& Hogan, 2010; Meyer, Stanley, Herscovitch, \& Topolnytsky, 2002).

Dada a importância de se compreender o impacto das mudanças na dinâmica das organizações, o presente trabalho tem por objetivo compreender como a resistência à mudança afeta o comprometimento dos trabalhadores, identificando também as principais fontes de resistência. $\mathrm{O}$ processo de mudança abordado neste estudo é a implantação da Avaliação de Desempenho Individual (ADI) pelo governo de Minas Gerais. É importante avaliar o processo de mudança no setor público porque, apesar de constituir-se de forma mais lenta e gradativa devido à necessidade de concordância de vários agentes da sociedade (Osborne \& Gaebler, 1994), faz-se extremamente necessário frente à demanda de maior qualidade e modernização nos serviços prestados.

\section{Mudanças no Setor Público: Inovações na Gestão de Pessoas}

Para qualquer empresa, pública ou privada, uma força de trabalho bem estruturada constitui uma variável fundamental para manter o bom desempenho organizacional. A gestão de pessoas no setor público, segundo Marconi (2003), sempre se pautou pela realização de atividades operacionais e pelo comportamento reativo. No entanto, tem-se modificado, na medida em que os gestores públicos estão mais atentos à importância dos investimentos na formação de servidores mais competentes e capazes de realizar as tarefas inerentes a cada organização.

O governo de Minas Gerais implantou o projeto Choque de Gestão, que objetiva melhorar a qualidade dos serviços públicos e reduzir seus custos, a partir da reorganização do arranjo institucional, da mudança de foco do papel do Estado e da modernização da gestão de recursos humanos. Tendo em vista a necessidade de estabelecer um novo padrão de administração de recursos humanos e a importância dos servidores públicos para o alcance do objetivo de implantar um modelo de gestão pública mais eficiente, os idealizadores do programa promoveram grandes mudanças na política de gestão de recursos humanos, com ênfase nos princípios meritocráticos e nos resultados.

Três mudanças na política de gestão de recursos humanos foram implementadas com grande impacto na vida dos servidores: implantação da avaliação de desempenho individual (ADI), reestruturação das carreiras; e realinhamento de competências. Procedeu-se, também, a reestruturação de todas as carreiras dos servidores ativos e inativos do estado. Esse programa envolveu 128 carreiras 
e aproximadamente 300 mil cargos, além da implantação de novas tabelas salariais (Vilhena, 2006). A avaliação de desempenho individual, ancorada no perfil de competências, passou a ser um requisito para movimentação na carreira.

O objetivo da implantação da ferramenta ADI é possibilitar o crescimento e o desenvolvimento dos servidores, bem como a melhoria da qualidade do serviço público (Vilhena, 2006). A avaliação é realizada por uma comissão de avaliação, formada pela chefia imediata do servidor, por colegas de trabalho e servidores da Unidade Setorial de Recursos Humanos. Para mensurar o desempenho dos servidores, foram definidos onze critérios avaliativos: qualidade do trabalho, produtividade no trabalho, iniciativa, presteza, aproveitamento em programas de capacitação, assiduidade, pontualidade, administração do tempo e tempestividade, uso adequado dos equipamentos e instalações de serviço, aproveitamento dos recursos e racionalização de processos, e capacidade de trabalho em equipe (Lei Complementar n. 71, 2003). Os conceitos obtidos na avaliação - excelente, bom, regular e insatisfatório - são registrados pela comissão de avaliação de acordo com o percentual alcançado pelo servidor (Lei Complementar n. 71, 2003).

Os resultados das avaliações, tanto dos gestores quanto dos não gestores, são utilizados para tomadas de decisões e atitudes em relação aos servidores, passando desde o cálculo do adicional de desempenho (ADE), promoções na carreira, até a perda do cargo. Dessa forma, a avaliação de desempenho individual como ferramenta de controle de gestão de pessoas pode trazer benefícios e consequências negativas para a vida profissional e pessoal do servidor. No entanto, as ameaças associadas à avaliação, juntamente à falta de clareza e à informação acerca do processo, parecem reais para os servidores, o que faz com que a resistência à implantação de instrumentos dessa natureza seja praticamente inevitável.

\section{Reações à mudança e ao comprometimento organizacional}

A mudança organizacional é definida como "qualquer transformação de natureza estrutural, estratégica, cultural, tecnológica, humana ou de qualquer outro componente, capaz de gerar impacto em partes ou no conjunto da organização" (Wood, Curado, \& Campos, 1994, p. 64). Entretanto, pesquisadores argumentam que qualquer mudança tem alto potencial para fracassar se os empregados não estiverem abertos para a mudança ou se eles acreditam que a mudança não será bem sucedida (Seijts \& Robert, 2011).

A resistência à mudança ocorre porque, em processos de mudanças, os indivíduos têm que sair de uma situação conhecida para uma situação desconhecida (Coghlan, 1993; Steinburg, 1992), tirando-os da zona de conforto habitual e estimulando uma força contrária no sentido de manter o status-quo (Nadler, 1987). Além disso, o gerenciamento de processos de mudanças com base em pressupostos tecnicistas sem levar em conta essa influência do elemento humano tem sido apontado por vários pesquisadores (Arendt, Landis, \& Meister, 1995; Dick, Ulrich, \& Tissington, 2006; Levine, 2001), como uma das principais causas de resistência e de fracassos de iniciativas de mudanças nas organizações. Outros pesquisadores argumentam que pouco é investido em comunicação, treinamento e acompanhamento da mudança propriamente dita resultando em desconfiança e resistência (Schneider \& Goldwasser, 1998). Enquanto os empregados esperam ser tratados com consideração e respeito durante processos de mudanças, ao contrário, os gestores que utilizam a coerção para obter a adesão à mudança geram falta de confiança e ressentimento com a gerência, culminando na rejeição parcial ou total da mudança pretendida (Hultman, 1995).

Pesquisas anteriores têm revelado uma grande diversidade de respostas individuais aos processos de mudança organizacional, que, em um continuum, podem assumir desde a forma de cooperação entusiástica até a de resistência ativa (Lines, 2005). Giangreco e Peccei (2005) constataram que o comportamento do indivíduo diante da mudança pode ser ativo ou passivo. Bovey e Hede (2001), em uma pesquisa envolvendo 615 respondentes de nove grandes empresas australianas em processo de grandes mudanças, concluíram que o comportamento individual pode ser de cooperação ou de resistência, ativo ou passivo, aberto ou fechado. 
Três fatores individuais e dois organizacionais têm dominado os estudos sobre as causas da resistência individual à mudança organizacional:

1. Indecisão e inconclusão: na mudança, o conhecido é substituído pelo incerto e ambíguo, gerando medo e insegurança (Coghlan, 1993; Nadler, 1987; Nadler \& Tushman, 1989; Steinburg, 1992). A menos que os trabalhadores reconheçam e estimem a necessidade de mudança organizacional, qualquer investimento individual será no sentido de manter as coisas como elas estão, colocando-se como barreira à mudança (Oreg, 2006; Wanberg \& Banas, 2000).

2. Ameaça ao convívio social: muitas mudanças organizacionais, como a realocação de funções, ameaçam a integridade dos grupos de amizade, de onde provém uma importante fonte de reconhecimento social de muitos funcionários (Chreim, 2006; Nadler, 1987), causando grande impacto na identidade social do grupo (Dick et al., 2006). Quando o indivíduo percebe que as mudanças trazem modificações indesejáveis no ambiente social das organizações, seu comportamento será no sentido de resistir ou boicotar tais mudanças.

3. Pressão do grupo: mesmo que os indivíduos aceitem ou queiram mudar seu comportamento, o grupo pode agir como limitador. A ansiedade causada por essa ameaça gera grande desconforto no grupo, podendo tornar-se um sério fator de resistência à mudança pretendida (Dick et al., 2006).

4. Consistência organizacional: as descrições de tarefas, regras e procedimentos originados nas formalizações devem ser rigorosamente seguidas pelos funcionários. Com isso, as ações são poderosamente determinadas por esta inércia estrutural (Hannan \& Freeman, 1984), que podem funcionar como uma barreira à mudança.

5. Experiências prévias: o receio quanto às experiências negativas anteriores pode criar atitudes de rejeição, resistência, bloqueios e rebeldia quanto à mudança. Armenakis e Bedain (1999) argumentam que a descrença ou recusa em acreditar que a organização pode ter sucesso na implementação de uma determinada mudança, mesmo havendo fracassado em momentos anteriores, pode ser um forte fator de resistência.

Pesquisas recentes sugerem que a resistência à mudança, afeta negativamente o comprometimento (Fedor et al., 2006; G. E. Lambert \& Hogan, 2010; Marques et al., 2007). Diferentes formas de comprometimento organizacional têm sido identificadas. No entanto, a tipologia mais utilizada na literatura é a desenvolvida por Meyer e Allen (1997), que integra três dimensões do comprometimento - afetivo, instrumental e normativo. $\mathrm{O}$ comprometimento afetivo indica forte envolvimento emocional do indivíduo com a organização, no qual ele deseja dar algo de si próprio para o bem-estar da organização (Meyer \& Allen, 1997; Mowday, Porter, \& Steers, 1982). O comprometimento instrumental é resultante da percepção que o indivíduo tem a respeito da relação custo e benefício, como integrante da organização (Becker, 1960). O comprometimento normativo é resultado da totalidade de pressões normativas internalizadas pelo indivíduo para que este se comporte de acordo com os objetivos e interesses da organização. Essa forma de comprometimento se desenvolve, em grande parte, pelo processo de socialização (Ajzen \& Fishbein, 1980).

Peccei, Giangreco e Sebastiano (2011) concluíram que os empregados que percebem a mudança como positiva tendem a ser mais comprometidos, dando suporte às conclusões de Fedor, Caldwell e Herold (2006) de que empregados que percebem a mudança como justa, necessária e benéfica para si mesmos e para a organização são mais comprometidos. Judge, Higgins, Thoresen e Barrick (1999) pesquisaram a relação entre autoconceito e tolerância ao risco, e a capacidade do indivíduo para lidar com mudanças. Os resultados deste estudo revelam que esses fatores são positivamente relacionados com a capacidade de lidar com mudanças, e que também afetam o comprometimento organizacional.

O novo contexto gerado pelas mudanças é percebido como improvável de satisfazer às aspirações e desejos do indivíduo, abalando o vínculo estabelecido com a organização antes da implementação da mudança. Portanto, nesta pesquisa, o pressuposto é que a resistência à mudança tem uma relação direta negativa com o comprometimento. Ou seja, quanto maior a resistência à mudança, menor será o comprometimento. Este argumento se justifica porque, segundo Meyer e Allen (1997), 
para reduzir a dissonância cognitiva, os indivíduos tendem a ver seu ambiente de forma que ele seja consistente com suas crenças e experiências anteriores. Assim, pode-se inferir que indivíduos que rejeitam as mudanças, ou o novo contexto gerado, tenderão a reduzir suas formas de vínculo com a organização, para minimizar o desconforto que surge com a dissonância entre o novo contexto e seus valores e experiências anteriores.

\section{Metodologia}

A natureza da pesquisa é explicativa e utilizou-se o método survey para a coleta de dados. A população pesquisada totaliza 141.164 servidores alocados em três secretarias de Estado: Secretaria de Estado da Educação (SEE), Secretaria de Estado da Saúde, especificamente as unidades da Fundação Hospitalar do Estado de Minas Gerais (FHEMIG), e Secretaria de Planejamento e Gestão (SEPLAG). Para garantir que os servidores selecionados representassem a população de cada órgão envolvido na pesquisa, adotou-se o processo de amostragem aleatória estratificada por secretaria. Segundo fórmula de Barnett (1991), a amostra calculada para a pesquisa, com erro amostral de 5\%, alcançou o índice mínimo de 196 indivíduos. A amostra obtida compõe-se de 679 respondentes, número muito superior ao valor mínimo sugerido. Os servidores participantes foram distribuídos da seguinte forma: 262 na Secretaria de Estado da Educação, 202 na Secretaria de Estado do Planejamento e Gestão, e 215 na Secretaria de Estado da Saúde.

A pesquisa trabalhou com dados quantitativos coletados a partir de um survey por meio de um questionário padronizado. De acordo com Selltiz, Wrightsman, e Cook (1987), o uso de questionário possui três vantagens: (a) custo baixo, uma vez que consiste em um instrumento que pode ser enviado pelo correio ou, simultaneamente, entregue a um grande número de informantes. (b) oferece a possibilidade de evitar potenciais vieses do entrevistador, pois o entrevistado tem a liberdade de responder às questões sem a presença intimidadora de outra pessoa (Cervo \& Bervian, 1983); e (c) os informantes poderão se sentir mais seguros em função do anonimato, o que lhes permite "se sentirem mais à vontade para expressar pontos de vista que temam colocá-los em situação problemática ou que julguem não ter aprovação" (Selltiz, Wrightsman, \& Cook, 1987, p. 17).

\section{Instrumento da pesquisa}

O questionário desenvolvido para esta pesquisa é composto de 3 partes. A parte 1 identifica os dados demográficos e hábitos sociais dos respondentes. A parte 2 avalia o construto comprometimento organizacional, usando o modelo de análise de Allen e Meyer (1990) e Meyer e Allen (1991, 1997), revalidado no Brasil por Bandeira, Marques e Veiga (2000). Finalmente, a parte 3 avalia o grau de aceitação e resistência dos pesquisados em relação à implantação da avaliação de desempenho individual pelo governo de Minas Gerais, adaptada do Inventário de Medida de Resistência à Mudança, desenvolvido e validado por Marques e Borges (2011). Esta escala é estruturada em cinco variáveis: indecisão e inconclusão, ameaça ao convívio social, pressão do grupo, consistência organizacional e experiências prévias com a mudança. As perguntas foram adaptadas para que os respondentes entendessem que a mudança organizacional ocorrida era, na verdade, a implantação da avaliação de desempenho individual. Excetuando as questões referentes aos dados funcionais, todas as demais foram obtidas por meio de escala do tipo Likert de seis pontos, tratadas como intervalares. Alguns autores, como Byrne (1995), afirmam que os problemas potenciais advindos do uso de variáveis ordinais como intervalares podem ser minimizados com o uso de cinco ou mais categorias.

A avaliação das variáveis desta pesquisa foi realizada por meio da análise fatorial, em que foram verificados os valores da medida de Kaiser-Meyer-Olkin (KMO), do teste de esfericidade de Bartlett, do alfa de Cronbach e a variância explicada pelo fator. Para avaliar a unidimensionalidade, verificouse, inicialmente, a adequação da amostra, utilizando a medida (KMO) e o teste de esfericidade de Bartlett. Na medida KMO, que mede a adequação da amostra, valores baixos indicam que a correlação entre os pares de variáveis não pode ser explicada por outras variáveis, e que o emprego da análise 
fatorial não é recomendável. Um valor superior a 0,5 é suficiente para afirmar que a correlação entre os pares de variáveis pode ser explicada por outras variáveis (Malhotra, 2001). Outra medida utilizada foi o teste de esfericidade de Bartlett, indicando também a adequação da amostra para a utilização na análise fatorial quando o valor $p$ é inferior a 0,05 .

Para avaliar a consistência interna de cada uma das variáveis, verificaram-se sua confiabilidade e sua unidimensionalidade. A confiabilidade foi avaliada segundo o alfa de Cronbach, cujos limites variam de zero a um. Quanto mais próximo de um, maior a indicação de confiabilidade da variável. A sugestão de Malhotra (2001) é que esses valores devem ser superiores a 0,60. Quanto à variância explicada do fator, segundo Hair, Anderson, Tatham e Black (2005), seu valor deve ser preferencialmente acima de 0,50 ou $50 \%$.

\section{Análise de dados}

Seguindo a abordagem de Hair et al. (2005), a análise quantitativa dos dados deu-se em várias etapas. Primeiramente, contemplou os dados ausentes e os valores extremos, com o objetivo de melhor conhecer os dados e, assim, trabalhá-los de forma a garantir que as análises seguintes fossem mais precisas e confiáveis. Em seguida, alguns pressupostos foram avaliados, como o de normalidade e o de linearidade.

O instrumento de avaliação utilizado nesta pesquisa foi estruturado em escalas multi-item tipo Likert com seis categorias, por meio das quais cada item é avaliado segundo uma conceituação específica. Os dados coletados via questionário sofreram tratamentos estatísticos distintos, por meio do pacote estatístico SPSS. Inicialmente, foram feitas análises baseadas em estatística descritiva e análise fatorial para avaliar a escala utilizada.

O método PLS (Partial Least Squares) foi utilizado para avaliar o modelo de resistência à mudança, ou seja, para identificar as principais causas da resistência à mudança organizacional. Este método é adequado para estimar a relação entre as variáveis, na medida em que considera o erro de estimação (Chin, 1998). Para identificar as causas da resistência à mudança, utilizou-se o software SmartPLS versão 2.0M3. Além do algoritmo PLS, o método bootstrap de reamostragem foi usado, para determinar o nível de significância dos caminhos (paths) identificados no modelo de resistência à mudança organizacional. Empregou-se ainda a análise de variância (ANOVA) para averiguar possíveis diferenças entre as três secretarias do estado de Minas Gerais. Finalmente, realizou-se ainda a análise de regressão linear após a análise de correlação para investigar o impacto da resistência à mudança como variável preditora do comprometimento organizacional.

Itens específicos, como os construtos e seus respectivos indicadores, separados pelas variáveis correspondentes, também foram descritos. Índices como média, desvio-padrão e porcentagem dessa frequência também seguem na pesquisa, bem como os resultados estatísticos das análises realizadas. Explicitados os procedimentos metodológicos, dedica-se a próxima seção à análise dos dados e à discussão dos resultados.

\section{Resultados}

Após a análise do modelo de medição, investiga-se a distribuição dos servidores em relação aos componentes do construto comprometimento organizacional: afetivo, normativo e instrumental. Por último, promove-se a análise da aceitação ou resistência dos servidores às mudanças implantadas nas instituições em que atuam, especialmente quanto à implantação da ADI e seus impactos no comprometimento organizacional. 


\section{Avaliação do instrumento de medição}

A medida KMO e o teste de esfericidade de Bartlett indicaram a possibilidade de uso da análise fatorial para a escala, pois para o indicador comprometimento afetivo $(K M O=0,85)$, para o comprometimento normativo $(K M O=0,82)$, e para o comprometimento instrumental $(K M O=0,67)$, os valores excederam 0,05. Foi possível então, reduzir a escala nas três dimensões do comprometimento organizacional. O próximo passo para a análise da escala compreendeu a obtenção do alfa de Cronbach, a realização da avaliação da unidimensionalidade dos indicadores e a apuração da variação explicada pelo fator obtido.

Para o indicador comprometimento afetivo, obteve-se uma variância explicada de 0,615 , ou seja, $61,5 \%$ da variabilidade da variável é explicada pelos itens que a representam. Obteve-se ainda uma consistência interna de 0,841, medida por meio do alfa de Cronbach. Os resultados indicam que, para as demais dimensões - normativa e instrumental - explica-se $60,73 \%$ e $63,72 \%$, da variância com consistência interna de 0,784 e 0,715 respectivamente. Os índices obtidos são considerados satisfatórios, segundo Hair et al. (2005).

A avaliação da escala de medida do grau de resistência à mudança indica a possibilidade de uso da análise fatorial para as cinco variáveis - indecisão e inconclusão, ameaça ao convívio social, pressão do grupo, experiência anterior e consistência organizacional - no construto resistência à mudança $(\mathrm{KMO}=0.67)$. Os resultados indicam ainda que as variáveis explicam $62,86 \%$ da variância do construto e revelam uma boa consistência interna, pois o alfa de Cronbach obtido foi 0,80.

\section{Análise de comprometimento e resistência dos servidores}

Para analisar o comprometimento dos servidores, primeiramente, calculou-se a média das respostas para cada uma das três dimensões analisadas: comprometimento afetivo, comprometimento normativo e comprometimento instrumental. Definiu-se também a variável comprometimento global, cujo indicador foi calculado com base na média das médias obtidas para três aspectos do comprometimento - afetivo, normativo e instrumental. Essa variável representa, portanto, o construto comprometimento organizacional.

Cada dimensão do comprometimento foi também analisada seguindo o seguinte critério: o nível de comprometimento é considerado baixo ou insatisfatório para valores médios até 2,99; entre 3,00 e 3,99 o nível de comprometimento é considerado normal ou satisfatório; finalmente, o nível de comprometimento organizacional é considerado elevado para valores médios acima de 4,00. A Tabela 1 mostra os resultados do comprometimento organizacional dos funcionários públicos segundo o critério especificado, informando também a média total e o desvio padrão para o construto comprometimento e suas dimensões.

Os resultados indicam que os servidores estão comprometidos principalmente por motivos afetivos $(M=4,17, D . P .=1,02)$, pois $66 \%$ da amostra percebem que a relação com a organização onde trabalham é caracterizada por razões emocionais. No entanto, foram obtidos ainda índices elevados de comprometimento instrumental $(M=3,82, D . P .=1,20)$, revelando que os respondentes mantêm o vínculo com a organização porque realmente necessitam, como afirmou 52,9\% da amostra. 
Tabela 1

Análise Descritiva dos Indicadores de Comprometimento

\begin{tabular}{lcccccccc}
\hline & & & \multicolumn{2}{c}{ Baixo } & \multicolumn{2}{c}{ Moderado } & \multicolumn{2}{c}{ Alto } \\
\cline { 4 - 9 } & Média & D.P. & \multicolumn{2}{c}{ Frequência } & \multicolumn{2}{c}{ Frequência } & \multicolumn{2}{c}{ Frequência } \\
\cline { 2 - 9 } Indicador avaliado & & & Abs. & Rel. (\%) & Abs. & Rel. (\%) & Abs. & Rel. (\%) \\
\hline Comp. afetivo & 4,17 & 1,02 & 84 & 12,4 & 147 & 21,6 & 448 & 66,0 \\
Comp. instrumental & 3,82 & 1,20 & 140 & 20,6 & 180 & 26,5 & 359 & 52,9 \\
Comp. normativo & 3,37 & 1,13 & 233 & 34,3 & 206 & 30,3 & 540 & 35,3 \\
Comp. global & 3,78 & 0,82 & 121 & 17,8 & 259 & 38,1 & 299 & 44,0 \\
\hline
\end{tabular}

Para aprofundar o entendimento do comprometimento organizacional nas instituições pesquisadas, procedeu-se ao teste de comparação de médias realizado por meio da análise de variância (ANOVA). Como resultado, constatou-se que existe diferença entre as organizações $[F(2,676)=6,60$; $p<0,01]$. A análise a posteriori revelou que o nível de comprometimento não difere entre as Secretarias do Planejamento $(M=3,89)$ e Saúde, representada pela Fundação Hospitalar - FHEMIG $(M=3,86)$. No entanto, o grau de comprometimento com a organização é significativamente diferente para a Secretaria da Educação, cujos resultados foram inferiores $(M=3,64)$.

Em relação ao construto resistência à mudança, os resultados sugerem que apesar das mudanças ocorridas na administração do estado de Minas Gerais, os níveis de resistência à implantação da avaliação de desempenho foram moderados $(M=3,85, D . P .=1,19)$. A análise de variância indica que não existe diferença nos níveis de resistência à mudança organizacional entre as três secretarias estudadas $[F(2,231)=1,529 ; p=0,22]$. Embora as diferenças não sejam significativas, observa-se que a Secretaria da Educação apresentou valores elevados de resistência $(M=4,02, D . P .=1,50)$ em comparação com as Secretarias de Planejamento $(M=3,64, D . P .=1,22)$ e Saúde $(M=3,78, D . P .=$ 1,45). Essa constatação corrobora para o entendimento de que existe uma relação entre resistência à mudança e ao comprometimento organizacional.

Finalmente, os resultados sobre resistência à mudança indicam que a principal causa de resistência à mudança são a indecisão e a inconclusão $(\lambda=0,66, t(679)=42,64, p<0,001)$, seguidas pela pressão do grupo $(\lambda=0,50, t(679)=28,41, p<0,001)$ e pela ameaça ao convívio social $(\lambda=$ $0,35, t(679)=6,09, p<0,001)$. Surpreendentemente, os fatores organizacionais foram considerados pelos respondentes como os aspectos que menos influenciam a resistência individual à mudança. As experiências prévias da organização com o processo de mudança $(\lambda=0,09, t(679)=6,39, p<0,001) \mathrm{e}$ a consistência organizacional diante das mudanças $(\lambda=0,05, t(679)=29,79, p<0,001)$ pouco impactam na decisão individual de resistir ou apoiar o processo de mudança nas instituições pesquisadas.

\section{Relações entre resistência à mudança e comprometimento organizacional}

Ao se analisar a percepção dos servidores acerca das mudanças introduzidas pelo governo de Minas Gerais por meio do Choque de Gestão e sua relação com o comprometimento, constatou-se que existe uma correlação linear e significativa, entre resistência à mudança e comprometimento organizacional $(r=-0,393, p<0,01)$. Esse resultado permite afirmar que há uma tendência de o comprometimento ser maior quando a visão em relação às mudanças organizacionais for mais positiva, ou seja, quanto menor o nível de resistência à mudança maior o comprometimento do trabalhador.

Essa relação se repete quando se avalia o grau de resistência à implantação da avaliação de desempenho individual pelo governo de Minas e as três dimensões do comprometimento. Há, portanto, uma correlação negativa e significante entre resistência à mudança e o nível de 
comprometimento afetivo $(r=-0,355, p<0,01)$, instrumental $(r=-0,103, p<0,01)$ e normativo $(r=-$ $0,408, p<0,01)$.

Para a análise do impacto que as mudanças promovidas com a implantação da avaliação de desempenho (ADI) promoveram no comprometimento global do servidor, prosseguiu-se à realização da regressão linear, em que a variável dependente é o comprometimento global apresentado pelo servidor, e a variável independente é o nível de resistência com a implantação da ADI. O resultado da análise de regressão sugere que a resistência à mudança influencia significativamente o nível de comprometimento do trabalhador. A Tabela 2 mostra os resultados da análise de regressão.

Tabela 2

Resultado da Análise de Regressão entre Comprometimento e Resistência à Mudança

\begin{tabular}{lccccc}
\cline { 2 - 5 } & \multicolumn{2}{c}{$\begin{array}{c}\text { Coeficientes não } \\
\text { padronizados }\end{array}$} & $\begin{array}{c}\text { Coeficientes } \\
\text { padronizados }\end{array}$ & \\
\hline Modelo & $\mathrm{B}$ & Desvio padrão & Beta & $\mathrm{t}$ & Sig. \\
Constante & 2,907 & 0,085 & & 34,329 & 0,000 \\
Resistência à mudança & 0,271 & 0,025 & 0,390 & 11,034 & 0,000 \\
\hline
\end{tabular}

Nota. Variável dependente: Comprometimento organizacional.

O coeficiente de determinação indica que $15,2 \%$ da variação nos níveis de comprometimento organizacional é explicada pelo grau de resistência à mudança do servidor público $\left(R^{2}=0,152\right)$, o que é considerado satisfatório para uma regressão simples. Com isso, pode-se afirmar que, a partir da amostra obtida, a hipótese de pesquisa foi confirmada, ou seja, os servidores que resistem à mudança organizacional tendem a apresentar níveis mais baixos de comprometimento com a organização do que os servidores que percebem as mudanças como positivas.

\section{Conclusões}

A partir dos resultados obtidos, foi possível chegar a algumas conclusões sobre o comprometimento e suas relações com a percepção que os servidores têm sobre as mudanças organizacionais implantadas pelo governo de Minas Gerais.

Primeiramente, 55,9\% dos servidores apresentam envolvimento moderado e baixo com suas instituições. Desses servidores, 17,8\% têm baixo comprometimento. Esse percentual representa 24.920 servidores nas três secretarias pesquisadas, sendo, portanto um número muito elevado. Apenas 44,0\% dos servidores localizam-se no nível alto de comprometimento. Ao se analisar o comprometimento daqueles que se julgam altamente comprometidos, constata-se que $66,0 \%$ estabelecem forte vínculo de natureza afetiva com as instituições em que trabalham. Mantêm vínculo fortemente ancorado na dimensão instrumental 52,9\% dos servidores. Considerando a definição de Meyer e Allen (1990), esses indivíduos estariam no serviço público e no cargo que ocupam porque precisam ou porque essa ainda é a melhor opção. Portanto, seu vínculo com a instituição é frágil. Para os demais 35,3\%, é forte o comprometimento normativo. Portanto, acham que devem permanecer na instituição por questões de identificação com seus objetivos. Ressalta-se que a distribuição dos servidores nas três dimensões do comprometimento não é excludente. Ou seja, um mesmo servidor pode estar simultaneamente comprometido nas três dimensões.

Em síntese, os servidores com forte comprometimento organizacional representam menos de $50 \%$ da amostra. Um contingente de 24.900 servidores está na categoria de pouco comprometido, e os demais apresentam comprometimento fraco. Esse resultado é preocupante, porque esses servidores 
têm alto potencial para apresentar comportamentos e atitudes que podem levar a baixo desempenho tanto em quantidade quanto em qualidade dos serviços prestados à sociedade. $\mathrm{O}$ comprometimento dos servidores está fortemente associado à necessidade do emprego e a aspectos afetivos desenvolvidos no local de trabalho. Aspectos relativos ao dever de cumprir os compromissos assumidos com a instituição têm baixo poder preditivo do comprometimento desses servidores.

No que tange à avaliação de desempenho individual, é elevada a média dos servidores que tendem a resistir à mudança proposta. Os servidores ainda não possuem uma visão clara dos seus impactos e potencialidades, e não acreditam que o programa de avaliação de desempenho individual possa realmente contribuir para melhorar o desempenho individual e/ou institucional. Esses dados apontam uma provável falta de participação dos servidores na fase de concepção e implantação do programa, bem como na realização de um processo de divulgação que não alcançou de modo abrangente e suficiente os servidores.

Eu relação às causas da resistência à mudança, os resultados deste estudo apontam para a indecisão e inconclusão com o processo de mudança como o principal fator. Isso significa que, quando o indivíduo ainda não tem certeza e está indeciso em relação às vantagens da mudança, ele tornar-se-á resistente, neste caso, à avaliação de desempenho, até que o funcionário perceba as modificações como benéficas para si e para e organização. A segunda causa da resistência individual à mudança identificada nesta pesquisa é a pressão do grupo. Neste sentido, o indivíduo se sente desencorajado pelos colegas de trabalho a aceitar as inovações propostas ou até mesmo a mudar suas práticas de trabalho. A ameaça ao convívio social é a terceiro fator identificado como fonte de resistência à mudança organizacional. O funcionário resiste porque, em decorrência da mudança, pode ter que deixar o grupo de trabalho atual ou ainda ter que conviver com colegas de trabalho que não lhe são simpáticos ou agradáveis.

Os resultados desta pesquisa indicam que as fontes individuais de resistência à mudança organizacional são significativamente superiores às fontes organizacionais. Com isso, o histórico organizacional de experiências prévias malsucedidas com processos de mudança também influencia a decisão individual de resistir à mudança, porém com menor intensidade. Outro fator de menor peso na decisão individual é a consistência organizacional. Quando o funcionário percebe que a mudança proposta não recebe apoio dos superiores, não apresenta coerência de conceitos e métodos, e não foi concebida por profissionais competente, sua reação é de indiferença ou resistência.

Diante disso, cabe ao Estado estabelecer estratégias que possam dar maior visibilidade ao projeto de ADI para os servidores. Conhecer as causas de descontentamento e resistências a essa ferramenta de gestão de pessoas é essencial para que o governo alcance efetivamente os objetivos primários e secundários referentes à gestão de pessoas no serviço público. Especificamente para o governo do estado de Minas Gerais, este estudo aponta que a Secretaria de Educação merece especial atenção porque apresentou índices elevados de resistência à implantação da avaliação de desempenho individual em comparação às outras secretarias estudadas.

Finalmente, o objetivo do trabalho é avaliar se indivíduos que resistem às mudanças tendem a apresentar níveis de comprometimento mais baixos, identificando as causas da resistência à mudança. Constatou-se que, quanto maiores os níveis de resistência à mudança organizacional, menor o grau de comprometimento do servidor com a organização. Isto é, quanto mais o servidor rejeita a mudança na organização, menos comprometido ele se torna. Este estudo identificou também os fatores individuais indecisão e inconclusão, pressão do grupo e ameaça ao convívio social como as principais causas da resistência individual à mudança organizacional. Por outro lado, uma variação positiva da aceitação e cooperação com a implantação da avaliação de desempenho individual resulta em um aumento do comprometimento global percebido. Ou seja, quem percebe a mudança positivamente tende a apresentar maior comprometimento com a organização.

Do ponto de vista acadêmico, o presente trabalho reforça pesquisas anteriores, como a de Peccei et al. (2011), que constataram que, tal como em estudos anteriores ao deles, a resistência é que interfere no comprometimento, e não o contrário, e também que empregados que percebem a mudança 
como positiva tendem a ser mais comprometidos. A presente pesquisa dá suporte também às conclusões de Fedor et al. (2006) de que empregados que percebem a mudança como justa, necessária e benéfica para si e para a organização, tendem a ser mais comprometidos.

Os achados desta pesquisa estão em consonância com as suposições de Wanberg e Banas (2000) e Oreg (2006), que reconhecem a indecisão individual como umas das principais barreiras à mudança. Dick, Ulrich e Tissington (2006) também haviam identificado a ansiedade gerada pela pressão do grupo como um grande fator de resistência à mudança pretendida, que coincidem com os resultados encontrados. E, finalmente, as conclusões de Nadler (1987) e Chreim (2006) em relação à importância da ameaça ao convívio social na decisão individual de cooperar ou resistir às mudanças, foram confirmadas neste estudo.

Acredita-se que, com base nesta pesquisa, o governo de Minas Gerais passa a dispor de subsídios consistentes para a validação das inovações implantadas e para a adequação, no que for viável, das políticas, das estratégias e dos procedimentos de gestão de pessoas no setor público, especialmente no que se refere ao programa de ADI, o que poderá contribuir para a melhoria do moral, da motivação, do desempenho, do comprometimento e da prestação de serviços de mais alta qualidade à sociedade brasileira.

Conclui-se, portanto, que os governos que irão ou estão passando por processos de mudança organizacional, sejam eles incrementais ou radicais, devem cuidar para que tais processos sejam bem conduzidos com o objetivo de diminuir os níveis de resistência individual. Esta pesquisa aponta que estratégias de gestão da mudança que focam em aspectos individuais tendem a obter mais sucesso do que estratégias focadas na excelência organizacional para elaborar e conduzir o processo de mudança. Com isso, o processo de mudança deve envolver psicologicamente o trabalhador (Elias, 2009), ser bem comunicada ao grupo (Lewis, 2006) e ser desenvolvido de tal forma que mantenha ou melhore a integração social no trabalho mesmo que envolva realocação de funções e reestruturação de equipes (Chreim, 2006).

Para as futuras pesquisas neste setor, será necessário introduzir novos métodos de coleta e análise de dados, além do método quantitativo. Como é sabido, nenhum método encerra em si mesmo todas as potencialidades de análise para todas as situações. É relevante aprofundar as pesquisas em resistência à mudança, levando em conta a análise de custos e benefícios das mudanças, da comunicação, da confiança e do estilo de atuação dos gestores para a estruturação da resistência dos servidores. É também imprescindível desenvolver projetos de pesquisa que tratem da gestão de pessoas no setor público como linhas de pesquisas permanentes, tendo em vista a complexidade de variáveis envolvidas neste setor.

\section{Referências}

Ajzen, I., \& Fishbein, M. (1980). Understanding attitude and predicting social behavior. Englewood Cliffs, NJ: Prentice-Hall.

Allen, N. J., \& Meyer, J. P. (1990). The measurement and antecedents of affective, continuance and normative commitment to the organization. Journal of Applied Psychology, 63(1), 1-18. doi: 10.1111/j.2044-8325.1990.tb00506.x

Arendt, C. H., Landis, R. M., \& Meister, A. B. (1995). Creating an organizational initiative that successfully addresses the human side of change. IIE Solutions, 27(5), 22-27.

Armenakis, A. A., \& Bedain, A. G. (1999). Organizational change: a review of theory and research in the 1990s. Journal of Management, 25(3), 293-315. doi: 10.1177/014920639902500303 
Bandeira, M. L., Marques, A. L., \& Veiga, R. T. (2000). As dimensões múltiplas do comprometimento organizacional: um estudo na ECT-MG. Revista de Administração Contemporânea, 4(2), 133157. doi: 10.1590/S1415-65552000000200008

Barnett, V. (1991). Sample survey: principles and methods. London: Arnold.

Bastos, A. V. B. (1995). Comprometimento organizacional: o estado da arte e uma possível agenda de pesquisa. Cadernos de Psicologia, 1(1), 44-63.

Becker, H. S. (1960). Notes on the concept of commitment. American Journal of Sociology, 66(1), 3240. doi: $10.1086 / 222820$

Bovey, W., \& Hede, A. (2001). Resistance to organizational change: the role of defence mechanisms. Journal of Managerial Psychology, 16(7), 534-548. doi: 10.1108/EUM0000000006166

Byrne, B. M. (1995). One application of structural equation modeling from two perspectives: explore EQS and Lisrel strategies. In R. H. Hoyle (Org.), Structural equation modeling: concept, issues and applications (pp. 138-157). London: Sage Publications Inc.

Cervo, A. L., \& Bervian, P. A. (1983). Metodologia científica: para uso dos estudantes universitários (3a ed.). São Paulo: McGraw-Hill.

Chin, W. W. (1998). The partial least squares approach to structural equation modeling. In G.A. Marcoulides (Org.). Modern methods for business research. Mahwah: Lawrence Erlbaum Associates.

Chreim, S. (2006). Managerial frames and institutional discourses of change: employee appropriation and resistance. Organizational Sudies, 27(9), 1261-1287. doi: 10.1177/0170840606064106

Coghlan, D. (1993). A person-centred approach to dealing with resistance to change. Leadership and Organization Development Journal, 14(4), 10-14. doi: 10.1108/01437739310039433

Dick, R. van, Ulrich, J., \& Tissington, P. A. (2006). Working under a black cloud: how to sustain organizational identification after a merger. British Journal of Management, 17(1), 69-79. doi: $10.1111 /$ j.1467-8551.2006.00479.x

Elias, S. M. (2009). Employees`commitment in times of change: assessing the importance of attitudes towards organizational change. Journal of Management, 35(1), 37-55. doi: $10.1177 / 0149206307308910$

Fedor, D. B., Caldwell, S., \& Herold, D. M. (2006). The effect of organizational changes on employee commitment: a multilevel investigation. Personnel Psychology, 56(1), 1-29. doi: 10.1111/j.1744-6570.2006.00852.x

Giangreco, A., \& Peccei, R. (2005). The nature and antecedents of middle manager resistance to change: evidence from the Italian context. International Journal of Human Resource Management, 16(10), 1812-1829. doi: 10.1080/09585190500298404

Hair, J. F., Anderson, R. E., Tatham, R. L., \& Black W. C. (2005). Análise multivariada de dados (5a ed.). Porto Alegre: Bookman.

Hannan, M. T., \& Freeman, J. (1984). Structural inertia and organizational change. American Sociological Review, 49(2), 149-164.

Hultman, K. E. (1995). Scaling the wall of resistance. Training and Development, 49(10), 15-18.

Jones, G. R. (2007). Organizational theory, design, and change (5a ed.). Upper Saddle River, New Jersey: Prentice Hal. 
Judge, T. A., Higgins, C. A., Thoresen, C. J., \& Barrick, M. R. (1999). The big five personality traits, general mental ability, and career success across the life span. Personnel Psychology, 52(3), 621-652. doi: 10.1111/j.1744-6570.1999.tb00174.x

Kotter, J. P. (1995). Leading change: why transformation efforts fail. Harward Business Review, 73(2), 59-67.

Lambert, E. I. (2006). Want to leave: a test of a model of turnover intent among correctional staff. Applied Psychology in Criminal Justice, 2(1), 57-83.

Lambert, G. E., \& Hogan, L. N. (2010). Wanting change: the relationship of perceptions of organizational innovation with correctional staff job stress, job satisfaction, and organizational commitment. Criminal Justice Police Review, 21(2), 160-184. doi: 10.1177/0887403409353166

Lei Complementar n. 71, de 30 de julho de 2003. (2003). Institui a avaliação periódica de desempenho individual, disciplina a perda de cargo público e de função pública por insuficiência de desempenho do servidor público estável e do detentor de função pública na administração pública direta, autárquica e fundacional do poder executivo e dá outras providências. Belo Horizonte, MG. Recuperado de http://hera.almg.gov.br/cgi-bin/nphbrs?co1=e\&d=NJMG\&p=1\&u=http://www.almg.gov.br/njmg/chama_pesquisa.asp \&SECT1=I MAGE \&SECT2=THESOFF $\&$ SECT3=PLUROFF $\&$ SECT6=HITIMG $\&$ SECT7 $=$ LINKON\&l=2 $0 \& \mathrm{r}=1 \& \mathrm{f}=\mathrm{G} \& \mathrm{~s} 1=\mathrm{LCP}$. TIPO.+e+71.NUME.+e+2003.ANO. \&SECT $8=$ SOCONS

Levine, G. (2001). Forging successful resistance. Bobbin, 39(1), 164-166.

Lewis, L. (2006). Employee perspectives on implementation communication as preditors of perceptions of success and resistance. Western Journal of Communication, 70(1), 23-46.

Lines, R. (2005). The structure and function of attitudes toward organizational change. Human Resource Development Review, 4(1), 8-32. doi: 10.1177/1534484304273818

Malhotra, N. (2001). Pesquisa de marketing: uma orientação aplicada (4a ed.). Porto Alegre: Bookman.

Marconi, N. (2003). A evolução do perfil da força de trabalho e das remunerações nos setores público e privado ao longo da década de 1990. Revista do Serviço Público, 54(1), 7-44.

Marques, A. L., \& Borges, R. S. G. (2011, novembro). Validando um modelo de resistência à mudança em organizações públicas. Anais do Encontro de Gestão de Pessoas e Relações de Trabalho, João Pessoa, PB, Brasil, 3.

Marques, A. L., Borges, R. S. G., \& Carvalho-Freitas, M. N. (2007, dezembro). Mudança organizacional: interferência nos indicadores de comprometimento e satisfação no trabalho. Anais do International Conference of the Iberoamerican Academy of Management, Santo Domingo, República Dominicana, 5.

Meyer, J. P., \& Allen, N. J. (1991). A three-component conceptualization of organizational commitment. Human Resource Management Review, 1(1), 61-89. doi: 10.1016/10534822(91)90011-Z

Meyer, J. P., \& Allen, N. J. (1997). Commitment in the workplace: theory, research and application. London: Sage Publications.

Meyer, J. P., Stanley, D. J., Herscovitch, L., \& Topolnytsky, L. (2002). Affective, continuance and normative commitment to the organization: a meta-analysis of antecedents, correlates, and consequences. Journal of Vocational Behaviour, 61(1), 20-52. doi: 10.1006/jvbe.2001.1842 
Mowday, R. T., Porter, L. W., \& Steers, R. M. (1982). Employee-organization linkages: the psychology of commitment, absenteeism and turnover. San Diego, CA: Academic Press.

Nadler, D. A. (1981). Managing organizational change: an integrative perspective. The Journal of Applied Behavioral Science, 17(2), 191-211. doi: 10.1177/002188638101700205

Nadler, D. A. (1987). The effective management of organizational change. In J. D. Lorsch (Ed.), Handbook of organizational behavior (pp. 358-369). Englewood Cliffs, NJ: Prentice Hall.

Nadler, D. A., \& Tushman, M. L. (1989). Organizational frame bending: principles for managing reorientation. Academy of Management Executive, 3(3), 194-204.

Oreg, S. (2006). Personality, context, and resistance to organizational change. European Journal of Work and Organizational Psychology, 15(1), 73-101. doi: 10.1080/13594320500451247

Osborne, D., \& Gaebler. (1994). Reinventando o governo: como o governo empreendedor está transformando o setor público. Brasília: MH Comunicação.

Peccei, R., Giangreco, A., \& Sebastiano, A. (2011). The role of organizational commitment in the analysis of resistance do change: co-predictor and moderator effects. Personnel Review, 40(2), 185-204. doi: 10.1108/00483481111106075

Schneider, D. M., \& Goldwasser, C. (1998). Be a model leader of change. Management Review, 87(3), 41-45.

Seijts, G. H., \& Roberts, M. (2011). The impact of employee perceptions on change in a municipal governement. Leadership \& Organization Development Journal, 32(2), 190-213. doi: $10.1108 / 01437731111113006$

Selltiz, C., Wrightsman, L. S., \& Cook, S. W. (1987). Métodos de pesquisa nas relações sociais (2a ed.). São Paulo: EPU.

Steinburg, C. (1992). Taking charge of change. Training and Development, 46(3), 26-32.

Vilhena, R. (2006). Rumo à segunda geração do choque de gestão. In R. Vilhena, H. F. Martins, C. Marini, \& T. B. Guimarães (Orgs.), O choque de gestão em Minas Gerais: políticas da gestão pública para o desenvolvimento (pp. 351-356). Belo Horizonte: Editora UFMG.

Wanberg, C., \& Banas, J. (2000). Predictors and outcomes of openness to changes in a reorganizing workplace. Journal of Applied Psychology, 85(1), 132-142.

Wood, T., Jr., Curado, I. B., \& Campos, H. M. (1994). Vencendo a crise: mudança organizacional na Rhodia Farma. Revista de Administração de Empresas, 34(5), 62-79. doi: 10.1590/S003475901994000500008 